\title{
Quand l'arabe « libanais » entre en classe (2010 à 2012)
}

Isabelle GRAPPE, Institut français du Liban

\section{Introduction}

De 2004 à 2010 j'ai été témoin en tant que conseillère pédagogique à 1'Institut français du Liban de demandes des écoles publiques et des bureaux pédagogiques privés pour l'accompagnement des enseignants dans la gestion du désintérêt dans les écoles francophones vis-à-vis de l'apprentissage des langues arabe et française de scolarisation, ainsi que des difficultés de compréhension et d'expression. La mise en place de la Recherche Action Formation (RAF) de 2010 à 2012, analysée dans cet article, a été menée par l'Institut français du Liban et en partenariat avec l'Université Saint Joseph et la directrice du Secrétariat des Ecoles Catholiques à leur demande. Ils ont exprimé la nécessité de développer des recherches dans les domaines de l'enseignement-apprentissage des langues qui accompagnent les acteurs de la formation à traduire les bases théoriques détenues dans les pratiques de classe.

L'analyse de besoins réalisée dans le cadre de cette RAF en novembre 2010 a montré que les enseignants de français et d'arabe classique cloisonnent l'enseignement des langues et interdisent systématiquement en classe le recours à l'arabe parlé ou courant utilisé dans les interactions socio familiales (famille, voisins, commerce, médias...) que nous appellerons « le libanais »( $c f$. partie 2.2 les langues dans la société libanaise).

La problématique dans cette Recherche Action Formation (2010-2012) est la suivante : dans quelle mesure la transgression par le recours au «libanais » peut permettre aux élèves de prendre conscience des ressources linguistiques qu'ils développent en dehors de l'école puis de les transférer en dehors de l'école pour les motiver à prendre la parole en classe d'arabe classique et de français?

Nous exposerons dans la première partie de cet article la complexité du paysage linguistique libanais : la tradition migratoire, les pratiques variées des langues en rapport avec les communautés existantes, le cloisonnement entre les langues apprises à l'école et le plurilinguisme des élèves dans leur vie quotidienne. La deuxième partie portera sur la recherche action menée et ses transgressions par rapport aux pratiques courantes d'enseignement des langues au Liban (cf. partie 2. 2 : le décalage entre le vécu de l'enfant et l'école). Nous décrirons les outils, la méthodologie, la démarche didactique des approches plurielles, le dispositif d'éveil aux langues (Evlang) ${ }^{1}$ et de didactique intégrée ${ }^{2}$ qui nous a permis d'agir sur l'attitude des enseignants et des élèves en classe pour mobiliser leurs 
attitudes et aptitudes insoupçonnées par l'école et les motiver pour prendre la parole en classe de français et d'arabe classique.

La troisième partie exposera les résultats. Dans la pré-enquête, le recours au « libanais » dans le questionnaire collectif et les entretiens individuels a confirmé que les élèves étaient passionnés par les langues en dehors de l'école. La réduction de l'enseignement des langues en classe au code ne permet pas aux élèves de mobiliser leur capital plurilingue et interculturel. Nous finirons par définir puis analyser l'impact de la démarche didactique des approches plurielles ainsi que le dispositif d'éveil aux langues et de didactique intégrée sur la nature de la prise de parole des élèves lors de l'activité orale en classe.

\section{Paysage linguistique libanais (Bassil, Isabelle Grappe : 2010)}

\section{1 Le Liban est un pays de vieille tradition migratoire}

Dès le dix-neuvième siècle, des Chrétiens maronites du Mont Liban émigrent vers les villes égyptiennes. D’autres Libanais choisissent le continent américain, du nord au sud, avant une troisième vague d'immigration qui amènera essentiellement des Musulmans chiites vers l'Afrique Noire francophone. A partir de 1950, des migrations définitives se feront vers le Canada et l'Australie ainsi que des migrations temporaires vers les pays pétroliers du Golfe et vers l'Europe occidentale qui prendront dès 1975 une ampleur considérable, au point de renverser la tendance qui faisait jusqu'alors du Liban une terre d'immigration plus que d'émigration. En effet les Arméniens se sont massivement réfugiés au Liban dès le début du siècle, suivis de façon plus lente mais continue par les Kurdes. Après la création de l'état d'Israël en 1948, ce sont les Palestiniens qui affluent et dans le même temps les Libanais d'Egypte et d'Afrique noire retournent nombreux au Liban, suivis entre 1958 et 1975 d'une vague d'émigration syrienne. En 1975 Beyrouth est devenue une agglomération refuge, accueillant les opposants aux régimes arabes autoritaires et les victimes de l'histoire dramatique du Proche-Orient. Mais la guerre a provoqué d'importants flux migratoires vers les pays du Golfe, l'Europe occidentale, le Canada et l'Australie : un tiers de la population a émigré, et il s'agit avant tout de ce qu'on a appelé «un exode des cerveaux » qui posera problème au pays après la guerre. Les nombreuses migrations internes dues à la guerre ont provoqué des regroupements communautaires recréant ainsi des régions confessionnellement très homogènes.

\section{I.2. Les communautés libanaises}

Les Musulmans : les Chiites sont maintenant les plus nombreux au Liban, devant les Sunnites pourtant majoritaires en général dans le Moyen-Orient. Les Druzes sont présents au 
centre du Mont Liban dès le dixième siècle. Alaouites et Ismaéliens forment une petite partie de la communauté musulmane.

Les Chrétiens : les Maronites sont les plus représentés au Liban, avec ensuite les Grecs orthodoxes, dont la présence est aussi très ancienne. Les Arméniens, orthodoxes, catholiques ou protestants ont toujours conservé une forte organisation communautaire et participent peu à la vie politique ; pendant la guerre, ils ont tenu à rester neutres. Grecs catholiques, Syriens orthodoxes ou jacobites, Syriens catholiques ou Syriens Chaldéens catholiques, orthodoxes ou nestoriens et Latins complètent la communauté chrétienne.

Les Israélites : il existait aussi à Beyrouth une petite minorité israélite.

C'est au seizième siècle qu'émerge, malgré les clivages innombrables des multiples communautés se partageant le territoire (Petit Liban), un émirat druze, vassal de la puissance ottomane, qui va nouer des relations avec les états occidentaux, principalement la France de François $1^{\text {er }}$ et les principautés italiennes, par le biais de commerçants, de religieux et d'enseignants. Au dix-neuvième, des affrontements entre Druzes et Maronites amèneront les Turcs à supprimer l'émirat. Les puissances occidentales saisiront ce prétexte pour manipuler chacune des communautés libanaises afin de servir leur intérêt qui est l'effondrement de l'empire ottoman. Elles feront ainsi du Liban le terrain privilégié de leurs rivalités de grandes puissances qui vont garantir à six l'autonomie d'un système politique original, né en 1864 et fondé sur la représentation des communautés religieuses. En 1920, la France obtient de la Société des Nations un Mandat sur les régions syriennes du Levant et proclame en septembre l'état du Grand Liban, transformé en république avec une constitution inspirée des lois françaises en mai 1926. La constitution entérine l'appartenance de chaque Libanais à une communauté religieuse représentée «équitablement» au sein de l'état par des députés élus suivant un double critère confessionnel et régional basé sur des équilibres démographiques officialisés en 1932 et jamais repris officiellement depuis. Après l'effondrement du régime de Vichy, entre 1941 et 1943 sous la pression des Britanniques et d'une insurrection nationale libanaise, l'indépendance se réalisera autour des grandes orientations d'un «pacte National »: équilibre entre Chrétienté et Islam; appartenance à la Nation Arabe et reconnaissance de la spécificité du pays et de ses relations anciennes avec l'Occident.

\section{I.3. Les langues dans la société libanaise}

L'arabe classique ou littéraire est la langue officielle du Liban. Il est utilisé à l'écrit essentiellement dans l'administration, la justice, l'armée, l'enseignement et les médias. Il sert également aux échanges politiques et commerciaux avec les pays arabes. C'est bien sûr la langue religieuse des Musulmans mais elle est aussi utilisée dans certaines pratiques 
catholiques (lecture des évangiles). A l'école, elle est enseignée et sert aussi de langue d'enseignement pour certaines matières. Elle est, en général, perçue comme une langue de prestige et de référence au monde arabe, ce qui ne va pas sans une certaine ambiguïté pour certains Chrétiens qui préfèrent se démarquer de ce qu'ils vivent comme la langue de la régression, «la langue des chameaux et du désert», bref, un arabe «lourd, brutal et sale » alors que les Musulmans, pratiquants ou non, y voient leur appartenance à la Nation Arabe. (Gueunier 177).

La langue parlée de la grande majorité de la population est ce que j'appellerai «le libanais », variété parlée de l'arabe, d'ailleurs spontanément appelée «arabe » par les Libanais. Elle est utilisée dans les médias à l'oral, dans le commerce, les échanges sociaux quotidiens et la famille. Elle est acquise de façon informelle en famille ou dans les échanges de la vie sociale et culturelle par la minorité arménienne notamment. Elle semble bénéficier actuellement d'une certaine généralisation de son usage dans les médias, l'enseignement et même la poésie (Bassil).

Le français s'est implanté au Liban avec le mandat français. Des écoles et universités chrétiennes (écoles des Saints Cœurs, écoles de la Sainte famille française, université Saint Joseph), des hôpitaux (Hôtel Dieu) assurent la diffusion de la langue parmi la population selon un triple critère de confession, de lieu de résidence et surtout de classe sociale (Abou). Si l'ensemble des Chrétiens (Arméniens compris) maîtrisent le français, nombre de Musulmans le parlent aussi, cette langue de la colonisation ayant été la langue du pouvoir et de l'accès à un marché du travail largement tourné vers l'étranger. Le français est largement présent en milieu urbain, dans les médias et dans la vie culturelle, ce qui en fait une langue seconde pour une grande partie des Libanais. C'est en outre parfois une langue «première » pour les Musulmans Chiites revenus d'Afrique francophone. Le français est enseigné dans les écoles privées payantes comme les lycées franco-libanais, les congrégations religieuses chrétiennes et certaines communautés religieuses musulmanes comme deuxième langue dès la maternelle. Il est aussi enseigné dans les écoles publiques gratuites du ministère de l'éducation nationale libanaise, dès le cycle primaire. Il sert de langue d'enseignement pour les matières scientifiques et la philosophie. Son intégration à la société libanaise est telle qu'elle peut lui conférer aux yeux de certains Libanais appartenant aux classes favorisées un statut de deuxième langue officielle ce qui n'est pourtant pas le cas actuellement. On trouvera ce cas à la fois chez l'interviewé libanais dans le cadre du mémoire de Véronique Laudet et dans le cadre de mon mémoire (2001). Le français est en tout cas perçu comme une langue de prestige pour sa supposée complexité, son histoire, sa culture et sa littérature abondante. Pour 
les femmes, il est le véhicule et le moyen d'une certaine libération, propre à rompre avec l'image de la femme musulmane soumise et analphabète. C'est aussi la langue de la science et de la modernité, avec de nombreux emprunts de termes techniques transférés après ajustements phonétiques à l'arabe. Pour certains Musulmans et Druzes, le français peut être ressenti comme un frein pour l'affirmation de l'identité arabe. C'est pour eux la langue snob des Chrétiens minoritaires mais dominants politiquement et économiquement et ils préconisent plutôt la normalisation de la langue sacrée du Coran. Avec les incertitudes de l'avenir de la reconstruction libanaise, les jeunes ont peut-être tendance à se défaire du lien privilégié avec le français qui deviendrait pour eux «une langue comme les autres» (Gueunier 176).

L'anglais s'est implanté avec les écoles protestantes installées par les Anglais présents en Syrie dans les régions musulmanes et particulièrement dans le Chouf où résident les Druzes. Les Etats-Unis ont également mis en place des universités (American University of Beyrouth (AUB), Notre Dame University (NDU), Lebanese Amercican University (LAU)) et un hôpital américain. De plus en plus l'anglais devient la langue professionnelle par excellence vers laquelle vont se tourner les garçons libanais alors que les filles, garantes de l'éducation des générations à venir, sont le plus souvent orientées vers le français par leurs parents. Langue d'enseignement dans les écoles protestantes au même titre que le français dans les écoles catholiques, l'anglais est aussi présent dans les médias. Recherché comme outil de promotion socio-économique, il est cependant perçu comme une langue facile, peu prestigieuse, voire un «pidgin» (Gueunier 176). Si l'usage de l'anglais se généralise au Liban comme ailleurs, il y est surtout présent au sein de la communauté musulmane.

L'arménien est bien sûr utilisé par la communauté arménienne dont il reste la langue de socialisation première même pour les générations nées au Liban. Les Arméniens acquièrent en général le libanais de façon informelle selon leurs besoins de communication ; ils maîtrisent souvent en plus le français et l'anglais, selon les relations qu'ils peuvent avoir avec la diaspora arménienne à travers le monde.

La nécessité du multilinguisme est la représentation sur les langues la mieux partagée au Liban. Il est vu depuis longtemps comme un enjeu essentiel pour l'avenir politique, commercial et économique du pays. A l'exception des classes sociales défavorisées, Musulmans résidant en zone rurale pour l'essentiel, la plupart des Libanais maîtrisent en effet une ou deux langues, voire davantage, en plus de l'arabe, oral et/ou classique.

La situation des deux variétés d'arabes mérite que l'on s'y arrête un moment : l'arabe classique peut sembler correspondre à la variété «haute» et l'arabe libanais à la variété 
«basse » des situations de diglossie décrites par Ferguson. De la même façon, le même arabe classique, langue officielle, peut être vu comme la langue légitime, dominante même si non perçue comme telle par les locuteurs, situation décrite par Bourdieu (27). A ces deux analyses, je préfère celle de Louis-Jean Calvet (229-242). En effet, l'arabe classique n'est pas véritablement parlé, au Liban pas plus qu'ailleurs, c'est une langue morte ou en passe de l'être, même si elle est lourde de représentations fortes (filiation avec les textes sacrés, relation avec l'idéologie panarabe et relation avec l'idéologie de la communauté des croyants musulmans). Cet arabe ne se transmet pas d'une génération à l'autre de façon naturelle, il est appris à l'école alors que le libanais est la langue de communication courante et se transmet de façon spontanée. D'autre part, au Liban comme dans bien d'autres pays arabes, «ce n'est pas la connaissance de l'arabe classique qui garantit un capital symbolique », ni l'accès au marché du travail, «mais plutôt celle du français et, dans une moindre mesure, de l'anglais », nous dit Calvet traduisant un extrait de Niloofar Haeri (238). L'arabe classique n'est alors légitime que sur un marché restreint (langue officielle et religieuse) alors que l'arabe «libanais» est la langue prédominante dans la vie quotidienne. Ce sont les langues étrangères (français et anglais) qui garantissent au Liban le capital symbolique et l'accès au marché du travail. Parce que l'arabe classique est immuable pour des raisons idéologiques et religieuses, il ne peut répondre à la demande sociale qui attend d'une langue officielle qu'elle remplisse des fonctions de modernisation et «d'européanisation ». La situation libanaise est d'autant plus ambiguë que la «valence» religieuse de l'arabe classique ne concerne pas la part chrétienne de la population libanaise, diminuant ainsi davantage le marché déjà restreint où la langue est légitime. Le terme de schizoglossie repris par Calvet à Einar Haugen (Calvet 229) me paraît un peu excessif quant aux effets qu'il suppose, et que l'interaction des attentes contradictoires de l'état, de la religion et des classes dominantes peut susciter. Pour autant, la description qu'il en fait me semble bien refléter la complexité de la configuration linguistique (représentations comprises) du Liban. Cette schizoglossie s'illustre notamment dans le fait que les Libanais ne nomment pas, ne distinguent pas spontanément les deux variétés, ce qu'ont montré les entretiens réalisés pour mon mémoire de DEA (Bassil 18).

\section{I.4. Le décalage entre le vécu de l'enfant et l'école}

Au Liban, dans les écoles francophones, le français jouit (ou souffre) d'un statut privilégié. Il faut enseigner la langue standard voire soutenue. Parler de langue de communication et de français oral commence dans certains établissements à faire son chemin. Mais dans beaucoup d'autres, il faut parler comme on écrit. L'enseignement du français de communication se heurte à sa peur de ne pas enseigner la langue «correctement » et de ne 
pas satisfaire aux exigences des examens officiels. L'enfant dans sa vie quotidienne est confronté au multilinguisme. Dans la rue, il peut lire des affiches en français, en arabe, en anglais. La télévision propose des films en version originale sous-titrée, les publicités sont souvent un mélange de publicités en français, anglais ou arabe. S'il va dans une librairie, il trouve tout un choix d'ouvrages multilingues. Les Libanais mélangent les langues dans la vie quotidienne alors qu'à l'école, on prend bien le soin de les séparer, de les cloisonner. L'enfant n'a alors pas de système de repère. L'école nie son «moi » social comme l'écrit Jouber, «l'école présente un système différent de l'extérieur. Il en découle que l'enfant traite alors les langues apprises à l'école comme des langues scolaires qu'il n'utilisera qu'à l'école. Il n'aura d'autre motivation que la motivation de satisfaire aux exigences des enseignants » (Courrier international 858).

\section{La RAF et ses transgressions}

Cette RAF a été menée dans une classe des écoles catholiques avec des élèves de 11 à 13 ans où les enseignants d'arabe classique et de français pensaient que les élèves étaient incapables de se concentrer et de participer à une activité scolaire. Lors de la pré-enquête en novembre 2011, le questionnaire à faire remplir aux élèves sur leurs pratiques plurilingues a été présenté en français par l'enseignante de français, en arabe classique par l'enseignante d'arabe et en «libanais » par moi. La possibilité de poser des questions, de répondre en « libanais » et d'alterner les langues a provoqué la participation de l'ensemble des élèves. Ils ne se sont pas contentés de poser des questions nuancées mais ont aussi suggéré de nouvelles rubriques. Cette présentation a poussé les enseignantes à demander le renouvellement de cette expérience en travaillant en doublette lors du dispositif mis en place dans la RAF. En effet, les élèves développaient inconsciemment en dehors de l'école dans leur vie quotidienne au Liban mais aussi dans leurs contacts avec la diaspora libanaise des répertoires langagiers très riches qu'ils adaptaient et nuançaient selon les interlocuteurs et la situation de communication (cf. résultats III.1). Nous avons alors décidé de donner les moyens aux enseignants d'exploiter ce bagage plurilingue et interculturel en classe. Les approches plurielles se sont avérées les plus appropriées puisqu'elles ne bannissent pas le recours à la langue première et ne traitent pas une langue et une culture particulière de façon isolée. Ces approches plurielles transgressent les principes de la didactique singulière et utilisent toutes sortes de variétés linguistiques de la famille, de l'environnement ...et du monde pour contextualiser l'apprentissage des langues Cadre de Référence pour les Approches Plurielles des langues et des cultures (CARAP 7- 8) ${ }^{3}$. Jacqueline Billiez ${ }^{4}$, consultante dans ce projet a présenté à 
l'équipe des conseillers pédagogiques et aux enseignants tous les outils existants pour faire travailler l'éveil aux langues. Notre choix s'est porté sur un dispositif d'Evlang ( $c f$. 2.3. La démarche d'Evlang), pour ses similarités avec la tradition migratoire des Libanais et le développement de leur plurilinguisme évolutif selon les situations et besoins de communication.

\section{II.1. Outils de collecte des données}

Les mêmes outils de collecte des données ont été mis en place en novembre et décembre 2010 avant la RAF et en 2011 lors de l'évaluation qualitative de l'impact du support contextualisé d'Evlang sur les élèves :

- un positionnement arabe libanais / classique et français niveaux A2/B1 en compréhension et production orales a été conçu selon le Cadre Européen Commun de Référence pour les Langues et les Cultures (CECR : 2001). ${ }^{5}$ Des adaptations ont été effectuées: les thématiques choisies ont pris en considération le contexte libanais. Les réponses étaient admises en «libanais » quand les élèves avaient du mal à s'exprimer en français et arabe classique, ce qui n'est pas le cas lors d'une passation traditionnelle.

- un questionnaire rempli par les 35 élèves sur leurs pratiques plurilingues: Les renseignements demandés ont porté sur les langues de communication des élèves dans leur environnement proche dans des situations de communication informelles (dans la cour de récréation de l'école avec leurs camarades de classe qui ont séjourné à l'étranger, pendant les activités extrascolaires, lors des loisirs, avec les voisins, les commerçants, les quartiers habités par différentes communautés libanaises comme les arméniens;...). D’autres questions ont porté sur les moyens de communication avec la diaspora libanaise (Skype, conversation téléphonique, SMS, Whats App...) et l'impact de ces relations sur leur façon d'être et de penser.

- des entretiens qualitatifs semi-directifs avec trois élèves perçus par les enseignantes d'arabe et de français comme «faible », «moyen » et «bon». Ces questions et réponses en « libanais » ont cherché à nous renseigner sur le vécu des élèves des cours de français et d'arabe classique avec les enseignants ainsi qu'à recueillir des suggestions pour rendre l'apprentissage des langues plus dynamique en classe. Ils ont aussi porté sur les centres d'intérêts, les loisirs et l'exposition aux langues des élèves les week-ends et pendant les vacances.

- sept observations de classe ont été filmées. Les séances 0 et 6 de compréhension et de production orales avant et après l'application du dispositif d'éveil aux langues et de didactique intégrée ont permis d'évaluer l'impact de l'expérimentation sur la pratique des 
enseignants. Les cinq autres séances ont permis d'analyser le processus mis en œuvre par le dispositif pour atteindre l'amélioration de la prise de parole des élèves dans les classes d'arabe classique et de français.

- un carnet de bord sur lequel j'ai noté toutes les micros transformations des élèves tout le long de la RAF dans les moments formels et informels.

\section{II.2. Méthodologie}

Le type d'analyse réalisée est qualitatif. Un dispositif d'éveil aux langues et de didactique intégrée a été adapté puis expérimenté en février, mars et avril 2011. Il a porté sur le support d'Evlang (Candelier) «I live in New York but... je suis né en Haïti ». Ce support didactique sur les parlers plurilingues a été conçu par une équipe du LIDILEM-Grenoble III, sous la direction de Billiez (Billiez et al).

\section{II.3 Les transgressions des objectifs généraux de la RAF}

A partir de ces constats, la RAF a expérimenté deux approches plurielles : l'éveil aux langues et la didactique intégrée (CARAP) ${ }^{6}$ dans une classe de $5^{\mathrm{e}}$ dont les élèves sont issus de classe défavorisée ou très moyenne. Par rapport aux pratiques pédagogiques des enseignantes d'arabe et de français avec lesquelles cette RAF a été effectuée, les objectifs listés ci-dessous sont hautement transgressifs. Ils agissent sur la posture des enseignants et visent à les réconcilier avec le « libanais » en leur donnant le moyen d'utiliser l'alternance codique ou le mélange des langues comme une stratégie d'apprentissage des langues de l'école :

- Concevoir que les élèves développent des ressources plurilingues non exploitées à l'école

- Permettre l'usage de toutes les composantes des répertoires communicatifs des élèves

- Valoriser les ressources plurilingues des élèves en classe au même titre que les langues de scolarisation

- Accepter que les stratégies d'apprentissage développées dans les langues de socialisation soient transférables pour mieux apprendre les langues de scolarisation

\section{La démarche de l'éveil aux langues (Cf. Evlang)}

Les objectifs que nous nous sommes fixés au Liban concernent la prise de conscience de la complexité et de la diversité des répertoires langagiers des locuteurs plurilingues dans leur entourage proche et éloigné. Ce support consiste également à faire prendre conscience aux élèves des combinaisons qu'ils font ou feront en tant que locuteurs plurilingues entre les différentes langues lors de leurs interactions dans la vie quotidienne avec les membres de leur entourage qui vivent au Liban ou à l'étranger. 


\section{La « logique du détour »}

La démarche d'Evlang est caractérisée par la logique du détour (Candelier 44). Celleci est considérée comme permettant une meilleure compréhension des phénomènes plurilingues grâce à la mise à distance que provoque la comparaison à d'autres langues et à d'autres fonctionnements linguistiques que ceux que vivent directement les élèves. Mais en permettant de faire entrer les élèves en résonance avec leur propre situation. En confrontant les élèves à plusieurs langues (français, anglais, créole, haïtien et espagnol), ce support didactique sensibilise à la diversité linguistique et légitime une langue minoritaire (le créole haïtien) dans le cadre scolaire.

«I live in New York»: Au cours de cette activité, les participants abordent le plurilinguisme en lisant un texte sur un adolescent de leur âge et les langues qu'il utilise à différents moments de sa vie, dans différents endroits et avec différentes personnes. Les participants complètent d'abord le sociogramme de ce personnage et font ensuite leur propre sociogramme (LIDILEM /Grenoble)

\section{Le contrat didactique}

C'est un contrat qui organise la gestion des interactions en classe, les stratégies des interactions puisque ces séances sont animées en doublette. Il s'est élaboré de la manière suivante :

«Les consignes seront données par l'enseignante de français en français et par l'enseignante d'arabe en arabe. Les réponses des élèves seront acceptées en français, en arabe libanais et en anglais. Elles seront reformulées en français par l'enseignante de français quand la consigne est donnée en français et en arabe classique par l'enseignante d'arabe quand la consigne est donnée en arabe classique. $»^{7}$

L'originalité de ce support réside au Liban dans sa mise en place auprès d'une classe d'élèves, dans leur majorité plurilingues. En effet, à la demande des deux enseignantes d'arabe et de français qui l'ont mis en œuvre en co-animation, il a été adapté par l'équipe de recherche sous la direction de Billiez pour qu'il soit utilisé comme support de didactique intégrée ( $c f$, annexe 3). Comme tous les autres supports d'Evlang, ce support s'articule selon trois étapes essentielles : la mise en situation ou ancrage, la situation-recherche, la synthèse (Candelier). Ces trois phases se sont étalées sur cinq séances auprès de la classe de 5ème qui a fait l'objet de notre étude. Nous avons choisi puis expérimenté en doublettes avec les enseignantes d'arabe et de français, à leur demande, cinq séances un support d'Evlang ${ }^{8}$ que nous avons remodelé dans une classe de $5^{\mathrm{e}}$ dans le but de proposer une formation de formateurs qui pourrait remédier à ce désintérêt des élèves. 


\section{Résultats}

Nous allons dans un premier temps constater le changement immédiat de l'attitude des élèves face à l'apprentissage des langues dès la pré-enquête. En effet, il s'est avéré que les élèves ont développé des ressources insoupçonnées par l'école dès la pré-enquête lors du questionnaire collectif et des entretiens individuels. Dans un deuxième temps nous allons analyser l'impact du dispositif sur la prise de parole des élèves en activité orale avant et après la mise en œuvre du dispositif.

\section{III.1. La transgression par le recours au « libanais » lors de la pré-enquête}

\section{- Le questionnaire collectif}

La formulation des questions en français et en arabe courant ainsi que la possibilité de répondre avec la langue de leur choix ont déclenché chez les élèves une participation active immédiate alors que ce n'est pas le cas pour l'apprentissage des langues de scolarisation. Le dépouillement du questionnaire collectif de départ des trente-cinq élèves sur les pratiques plurilingues des élèves a immédiatement dévoilé des ressources plurilingues développées grâce au contact avec la diaspora libanaise dans dix-sept pays et cultures ainsi qu'avec les employées de maison indiennes et chinoises.... Ils ont en effet développé un «éveil aux langues » et une motivation poussée pour l'apprentissage des langues en dehors de la classe comme à la télévision, avec les voisins, dans la rue :

«A la télévision je regarde en français en arabe classique et en anglais avec les sous-titres /j'aime aussi regarder/des films anglais doublé en arabe libanais de temps en temps avec mes parents/ je regarde les films indiens avec la bonne/ j'entends le chinois avec la bonne des voisins/ je regarde des chaines françaises »

«J'ai envie d'apprendre le latin, l'italien, le mexicain, le brésilien/le canadien/le turc /l'allemand/l'espagnol/le chinois /»

Ces témoignages rejoignent l'affirmation suivante : «La télévision est puissamment pluriculturelle (Abdallah et Porcher 165). La compétence interculturelle (CARAP) a aussi été développée grâce aux contacts par Skype avec des cousins scolarisés dans des cultures assez éloignées comme le Canada et l'Arabie Saoudite. Le témoignage suivant nous montre à titre d'exemple comment leur façon de percevoir et de faire face aux problèmes rencontrés a été modifiée :

«Quand je parle avec mes cousins au Canada et en Arabie Saoudite j'apprends comment ils pensent et ça m'aide à résoudre des problèmes avec mes parents et mes copains » 


\section{- La transgression par le recours au « libanais » lors des entretiens semi-directifs}

La légitimation du « libanais » lors des entretiens semi-directifs avec les élèves a aussi induit un changement immédiat de leur posture. Elle a débloqué l'expression des trois élèves et en particulier celle de l'élève considéré comme le plus faible. Ils ont exprimé pourquoi ils étaient «réfractaires » à l'apprentissage des langues en classe. L'élève considérée comme «moyenne » par les enseigantes a manifesté un sentiment d'insécurité linguistique lors de la production orale dans les langues de scolarisation en classe alors que ce n'est pas le cas en «libanais »:

I : Quelles langues aimez-vous parler ? dans quelle(s) langue(s) vous sentezvous à l'aise?»

C : Le libanais bien sûr/pas l'arabe de l'école/ en arabe classique, français et anglais j'ai peur de ne pas trouver les mots et de me tromper

L'élève considéré par les enseigantes comme «le plus faible » refuse la réduction de l'apprentissage des langues de scolarisation au code ainsi que le manque de lien entre les contenus scolaires et la vie professionnelle :

$\mathrm{J}$ : Moi ça ne m'intéresse pas d'apprendre uniquement la grammaire, la conjugaison, l'expression écrite et comment faire les liaisons. J'aimerais surtout parler la langue et écrire des lettres. Quand je chercherai du travail plus tard par exemple en Amérique, je ne vais pas dire à l'employeur j'ai/tu as /il a... Si je ne sais pas communiquer dans ces langues je ne pourrai pas trouver du travail.

Dans la vie de tous les jours il développe en regardant des émissions télévisées la capacité de s'approprier les connaissances en plusieurs langues selon des centres d'intérêt liés à sa classe d'âge :

J : L'école ne m'apprend pas aussi bien que la télévision/tout ce que je cherche je le trouve à la télévision mais pas à l'école/ par exemple comment on fabrique un avion/ ou sur la chaine animaux / on dit que l'animal n'est pas intelligent mais il m'apprend beaucoup de choses/ pour comprendre je regarde les images et les mouvements j'essaye de deviner ce qu'ils disent et après je lis les sous-titrages pour vérifier si j'ai bien compris

Il développe aussi des stratégies d'auto-apprentissage lors de ses activités extrascolaires comme les camps scouts :

J : Avec les scouts aussi c'est pas comme l'école /on doit survivre dans la nature et apprendre par nous-mêmes

«Le libanais » lui a aussi permis d'exprimer son désir de trouver du sens dans les apprentissages scolaires. Cette capacité à justifier son point de vue n'est pas apparue dans le test de positionnement effectué juste avant l'entretien semi-directif en arabe classique et en français : 
$\mathrm{J}$ : j'aimerais qu'ils changent le programme/qu'ils nous apprennent quelque chose de plus profond/ de plus philosophique

La transgression par le recours au « libanais » a fait prendre conscience aux élèves de leurs ressources plurilingues développées en dehors de l'école.

- L’impact du dispositif sur la prise de parole des élèves lors de l'activité orale en classe

Pour vérifier que cette transgression en libanais a eu un effet plus poussé grâce à l'application du dispositif d'éveil aux langues et de didactique intégrée dans les prises de parole en arabe classique et en français, nous allons commencer par faire une analyse des séances 0 filmées avant la RAF dans le but d'avoir une idée claire du niveau initial des élèves à l'oral en français et en arabe avant la mise en œuvre de ce dispositif. Nous nous appuierons par la suite sur une étude de cas représentative pour les comparer avec la séance 5 (dernière séance du dispositif) et la séance 6 filmée lors de séances de classes traditionnelles après le dispositif.

\section{- $\quad$ Séance 0 d'arabe classique}

Si nous cherchons à observer la nature des prises de parole des élèves en arabe classique, nous remarquons que dans la séance 0 la majorité des réponses sont sollicitées par l'enseignante puisqu'elle donne à chaque fois une phrase et demande aux élèves de la finir ou sollicite des productions mémorisées préalablement. En arabe 86/201 énoncés des élèves ont été réalisés en chœur et les 115 autres sont des prises de parole individuelles constituées de 1 à 7 mots maximum et seulement 2 énoncés contiennent entre 15 et 23 mots. L'énoncé 161 / suivant est représentatif de l'arabe très soutenu utilisé par l'enseignante dans l'ensemble de la séance d'arabe :

\begin{tabular}{|c|c|c|}
\hline 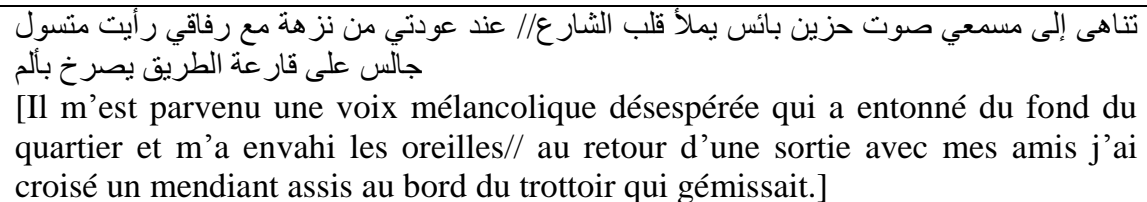 & ARC & تينا \\
\hline
\end{tabular}

La chercheure d'arabe faisant partie de l'équipe de la RAF, Siham Harb, spécialisée en littérature enfantine arabe, affirme que la langue utilisée dans cette séance appartient à un registre soutenu qui n'est pas adéquat au niveau des élèves de la classe de $5^{\mathrm{e}}$.

Les énoncés 15 et 16 suivants sont représentatifs des prises de parole individuelles et collectives tout au long de la séance. Dans l'énoncé 15, l'enseignante commence la phrase en disant «donc le pronom personnel désignant» puis s'arrête pour laisser les élèves finir la phrase par le mot «énonciateur» : 


\begin{tabular}{|c|c|c|c|}
\hline [donc le pronom personnel désignant] & Arc & غلاديس & 15 \\
\hline [L'énonciateur] & Arc & تلاميذ & 16 \\
\hline
\end{tabular}

Nous pouvons dire que tous les énoncés des élèves et de l'enseignante de cette séance ont été formulés en arabe classique. La seule prise de parole en «libanais » était sous forme humoristique et a été considérée comme une dérision et non pas comme un moyen d'apprentissage possible.

\section{- $\quad$ Séance 0 de français}

Nous retrouvons une similarité dans les séances d'arabe et de français dans le sens où la presque totalité des énoncés des élèves 96 / 98 sont sollicités par l'enseignante. Nous pouvons supposer que la différence de nombre de prise de parole collective est uniquement de 2/96 prises de parole individuelles en français par rapport à l'arabe est probablement due au registre de français courant utilisé ainsi qu'au thème «Les pays du Moyen Orient » de la séance plus relié aux centres d'intérêt des élèves :

\begin{tabular}{|l|l|l|l|}
\hline 11 & prof & $f r$ & $\begin{array}{l}\text { Donc on va se rappeler le thème quel thème nous travaillons alors nous } \\
\text { avons introduit le thème terre d'orient n'est pas on va se rappeler } \\
\text { ensemble le thème qu'on va introduire et j'écoute alors } x\end{array}$ \\
\hline 12 & elv & $f r$ & Palestine \\
\hline 13 & Prof & $f r$ & Palestine ok oui $x$ \\
\hline 14 & elv & $f r$ & l'Égypte \\
\hline
\end{tabular}

Cependant dans les énoncés ci-dessus, ce qui est similaire à la séance 0 d'arabe, ce sont les réponses sollicitées par l'enseignante. Elles sont majoritaires (92/98) et ont été apprises par cœur préalablement comme nous pouvons le remarquer dans l'énoncé 11 cidessus où l'enseignante demande aux élèves de «se rappeler » et de faire un listing des pays de la terre d'Orient comme dans les énoncés 12 les mots «Palestine » et 14 «Egypte».

\section{- $\quad$ Nature des réponses en français dans la séance pré-test (séance 0)}

A la différence de la séance d'arabe, l'énoncé 104 ci-dessous de 18 mots a été initié par l'élève avec une tentative de production personnelle est représentatif de 6 autres énoncés de 10 à 23 mots. La totalité de ces énoncés sont en lien avec les pratiques plurilingues des élèves :

\begin{tabular}{|c|l|l|l|}
\hline $\begin{array}{c}104 \\
\text { (18mots) }\end{array}$ & élève & Fr & $\begin{array}{l}\text { Les gens commencent à parler le français // les gens qui ne } \\
\text { savent pas le français maintenant ils savent }\end{array}$ \\
\hline
\end{tabular}

Dans 7 énoncés français les élèves ont alterné en « libanais » le temps d'un mot mais pour nommer des éléments reliés à la culture libanaise : la danse traditionnelle libanaise 
« dabké » comme dans les énoncés 20 et 23, la musique traditionnelle libanaise dans l'énoncé 29 «zaffé » et pour donner le nom de plats libanais «mezzé, taboulé et hommos » comme dans les énoncés 25 et 42 . Ces deux séances ont été filmées après la passation des questionnaires bilingues avec les enseignantes en français/libanais ou arabe classique /libanais et après la présentation de la RAF. L'enseignante de français a affirmé à la fin de l'observation de la séance 0 (carnet de bord) qu'elle avait été affectée par la participation des élèves lors du questionnaire de la pré-enquête et elle avait déjà modifié ses pratiques en acceptant l'alternance en «libanais » et en choisissant des thèmes proches de la culture des élèves, ce qui n'a pas été le cas de l'enseignante d'arabe.

\section{- $\quad$ Etude de cas représentative des résultats}

Le choix s'est porté sur l'apprenant 14 parce qu'il était perçu comme «le plus faible » par les enseignantes avant la mise en œuvre du dispositif. Cette étude de cas est d'autant plus intéressante que cet élève ne s'exprimait presque pas dans les premières séances : (1 à 2 énoncés). Ses prises de parole ne sont guère plus importantes dans les premières séances de français : 1 énoncé dans la séance 1 et aucune prise de parole dans les séances 2 et 3 en français. A la fin du dispositif il a participé davantage et activement pendant les séances d'oral. Ses interventions ont plus que triplé dans les 2 dernières séances observées : 17 énoncés dans la séance 5 (dernière séance du dispositif) et 24 énoncés dans la séance 6, séance d'arabe classique traditionnelle après la fin du dispositif dont 23 en arabe classique et 1 en arabe libanais ainsi que 22 prises de parole en français à la fin du dispositif dont 6 en libanais et 2 en franco-libanais.

\section{- Prises de parole initiées et énoncés représentatifs du développement de}

\section{l'activité orale des élèves}

Nous allons présenter quelques énoncés qui vont nous montrer comment l'activité orale s'est améliorée grâce à la transgression par le dispositif et le recours au libanais.

\section{- Enoncés représentatifs en classe d'arabe classique dans les séances 5 et 6}

Lors de la séance 6 au cours d'un travail sur une affiche sur laquelle est dessinée une cigarette qui fume et de la fumée tout autour de la planète, à la question de l'enseignante «que pensez-vous de cette affiche ? »l'apprenant 14 a donné son point de vue et l'a justifié en arabe classique en expliquant comment les habitants de la planète la polluent, brûlent les arbres et détruisent la couche d'ozone : 


\begin{tabular}{|c|c|c|c|}
\hline 725 & App 14 & $A R C$ & 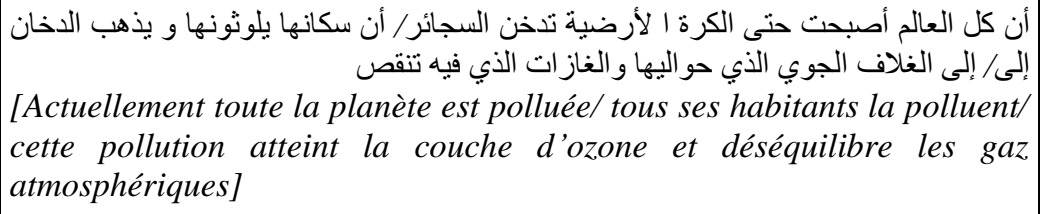 \\
\hline
\end{tabular}

\begin{tabular}{|c|c|c|c|c|}
\hline 203 & App14 & $F r$ & \multicolumn{2}{|c|}{ c.à.d. euh qui sont basées sur une autre langue } \\
\hline 204 & Ens & $F r$ & \multicolumn{2}{|l|}{ Exemple } \\
\hline 205 & App14 & $F \boldsymbol{F r}$ & \multicolumn{2}{|l|}{ En Haïti } \\
\hline 206 & Ens & & \multicolumn{2}{|c|}{ Le créole tu veux dire } \\
\hline 207 & App14 & $\boldsymbol{F r}$ & \multicolumn{2}{|c|}{$\begin{array}{l}\text { Oui il est proche du français ça veut dire comme famille comme chien c'est aussi chien } \\
\text { avec y }\end{array}$} \\
\hline 415 & \multicolumn{2}{|c|}{ App 14} & $A R C$ & $\begin{array}{l}\text { إلن حرق الغابات يؤدي إلى قلة الأوكسيجين عن سطح الأرض لأن الأشجار هي مصدر } \\
\text { [le fait de brûler les arbres engendre la diminution de l'oxygène dans } \\
\text { l'air et sur toute la planètel car les arbres sont la source même de } \\
\text { l'oxygène] }\end{array}$ \\
\hline
\end{tabular}

Il propose aussi des solutions pour remédier à la pollution en proposant de faire pousser des arbres sur les immeubles :

\begin{tabular}{|c|c|c|c|}
\hline 376 & App 14 & $A R C$ & 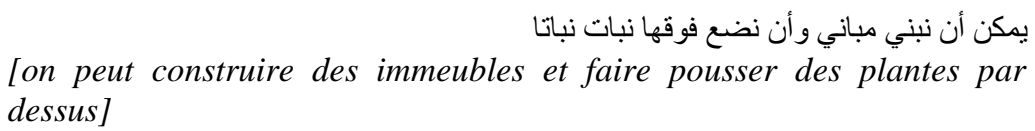 \\
\hline
\end{tabular}

- $\quad$ Enoncés représentatifs en classe de français dans les séances 5 et 6

L'apprenant $14 \mathrm{a}$ pu produire des phrases simples en français :

\begin{tabular}{|l|l|l|l|}
\hline 627 & Appl4 & Fr & comme quand on va à un camp \\
\hline
\end{tabular}

A la question de l'enseignante de français dans l'énoncé 200:

\begin{tabular}{|l|l|l|l|}
\hline 200 & San & Fr & $\begin{array}{l}\text { en ce qui concerne la dernière question Qu'est-ce qu'il faut } \\
\text { faire précisément? Qu'est-ce qui vous est demandé de faire? }\end{array}$ \\
\hline
\end{tabular}

L'apprenant 14 a pu reformuler, expliciter et exemplifier une consigne complexe sur l'intercompréhension entre les langues romanes à ses camarades en français dans les énoncés 201 à 209 :

Il a pu aussi transférer l'expérience de l'intercompréhension entre le créole et le français dans le dispositif dans sa pratique des langues en le comparant aux mots qu'il apprend en espagnol : et en italien en les comparant avec les mots français :

\begin{tabular}{|l|l|l|l|}
\hline 841 & App14 & Fr/italien & $\begin{array}{l}\text { Euh il ya les () on a appris autres langues () l'italien euh il y a } \\
\text { dorito c'est droit en français () il y a amigos dans l'espagnole } \\
\text { ca veut dire ami }\end{array}$ \\
\hline
\end{tabular}

Il a pu décrire, analyser, commenter et nuancer son expérience plurilingue en français. Il a ainsi exprimé son point de vue concernant l'importance du plurilinguisme pour l'épanouissement personnel, l'insertion sociale et une ouverture sur le monde : 


\begin{tabular}{|l|l|l|l|}
\hline 771 & App14 & Fr & dans la vie il y a un mélange de langue \\
\hline 772 & San & Fr & Oui dans la vie ? quelle vie ? c'est-à-dire \\
\hline 773 & App14 & Fr & Vie scolaire et vie/ autre \\
\hline 774 & San & $F r$ & Donc Donc à l'école et à l'extérieur de l'école oui \\
\hline 775 & App14 & $F r$ & Plusieurs langues \\
\hline 776 & San & $F r$ & Donc un mélange de langue \\
\hline 777 & App14 & $F r$ & $\begin{array}{l}\text { Euh il y a ça veut dire les gens /maintenant va euh a savoir } \\
\text { beaucoup de langues/ pour/ être /membre bien/ pour la société }\end{array}$ \\
\hline
\end{tabular}

L'apprenant 14 a pu transférer ce qu'il a appris dans une langue vers les autres. Même s'il peut prendre la parole en français, il continue à avoir recours au libanais pour nuancer et approfondir une nouvelle idée avant de la reformuler en français. Les énoncés 622 et 623 montrent à titre d'exemple comment il commence à développer l'idée de l'intérêt du plurilinguisme par la pratique non seulement du libanais mais aussi de l'anglais et du français en libanais. Il reformule ensuite en français dans l'énoncé 789 son idée de la nécessité de parler des langues étrangères pour s'ouvrir sur le monde :

\begin{tabular}{|c|c|c|c|}
\hline 622 & San & $F r$ & $\begin{array}{l}\text { euh aux scouts vous parlez libanais mais vous utilisez d'autre } \\
\text { mots }\end{array}$ \\
\hline 623 & App14 & Arl & [ on parle le libanais ensemble] \\
\hline 624 & San & $F r$ & Oui \\
\hline 625 & Appl4 & $A r l$ & $\begin{array}{r}\text { بس كمان منشتغل ع غبر لغات () بutres langues] } \\
\text { [on utilise aussi d'autres lan }\end{array}$ \\
\hline 626 & San & $\mathrm{Fr}$ & c.à.d comment \\
\hline 789 & App14 & $\mathrm{Fr} / \mathrm{Arl}$ & 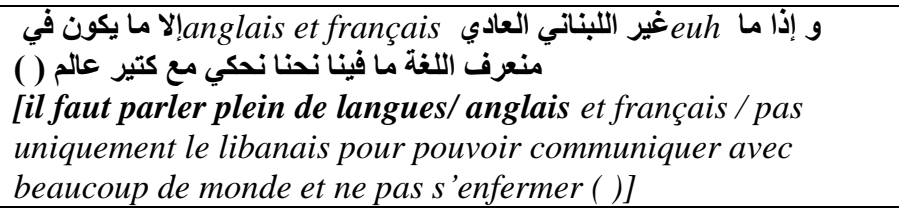 \\
\hline
\end{tabular}

\section{Conclusion}

Pour conclure, la transgression par le recours au libanais dans la préenquête a d'abord débloqué l'activité de production orale en «libanais » et a démontré que même les élèves considérés comme les plus «faibles » pouvaient argumenter en langue maternelle alors qu'ils ne pouvaient que compléter par des mots des phrases initiées par l'enseignante ou répéter des phrases mémorisées quand il leur arrivait de prendre la parole. Nous avons constaté dans les séances 5 et 6 que le dispositif a donné le moyen à l'élève 14 de transférer cette capacité de production orale en français et en arabe classique. Même si au fur et à mesure des séances du dispositif l'apprenant 14 ne choisit pas la facilité de s'exprimer systématiquement en «libanais » en classe, il y a recours pour nuancer, approfondir ou développer de nouveaux propos. L'étude de cas exposée plus haut montre que l'apprenant 14 est passé du niveau A2 
au niveau B1 selon les niveaux du CECR (27-28). Il a pu décrire un évènement, exprimer son opinion, justifier et expliquer son point de vue avec des phrases qui sont plus riches, plus longues et plus cohérentes (descripteurs CECR, 27-28). Ces résultats n'auraient pu être atteints sans la permission du recours au «libanais», la prise en compte des centres d'intérêts, des ressources spécifiques aux petits élèves libanais et l'application des approches plurielles qui correspondent au contexte libanais, ce qui pose la question de l'adaptation du CECR à tous les contextes.

Ces résultats peuvent être généralisés à l'ensemble des élèves de la classe. Dans la séance 6 les élèves ont effectué 37 énoncés en libanais /479 en arabe classique et 11 énoncés en libanais /354 en français.Ces résultats se recoupent en quelque sorte avec les propos suivants de Meirieu dans son article «apprentissage et transgression : «Le goût d'apprendre peut émerger dès lors que le maître propose des objets culturels, grâce auxquels chacun relie ce qui lui est le plus intime à ce qu'il y a de plus universel. »

\section{Bibliographie}

Bizri, Fida. Parlons arabe libanais. Paris : Harmattan, 2010.

Billiez, Jacqueline. «De la didactique des langues à la didactique du plurilinguisme ». Hommage à Louise Dabène. Grenoble : CDL-LIDILEM, 1998.

--- et Agnès Millet. «Représentations sociales : trajets théoriques et méthodologiques ». Les représentations des langues et de leur apprentissage, références, modèles, données et méthodes. Dir. Daniel Moore. Paris : Didier, 2001. 31-49.

--- et al. «I live in New York but... je suis ne en Haiti » : support didactique sur les parlers bilingues : P. SOCRATES/LINGUA. U Stendhal - Grenoble III, 1999.

Byram, Michael. Sociétés multiculturelles et individus pluriculturels : le projet de l'éducation interculturelle. Langues dans l'éducation/langues pour l'éducation. Strasbourg: Conseil de l'Europe, Division des politiques linguistiques. 2009.

Calvet, Louis-Jean. Pour une écologie des langues du monde. Paris : Plon, 1999.

Candelier, Michel. L'éveil aux langues à l'école primaire Evlang : bilan d'une innovation européenne. Bruxelles : De Boeck, 2003.

Council of Europe. «Council of Europe. Learning, Teaching, Assessment ». Strasbourg, Language Policy Unit, 2001. www.coe.int/lang/CEFR

Gueunier, Nicole. Le français du Liban : cent portraits linguistiques. Paris: Didier Erudition, 1993. 
---. «Je suis un Libanais typique : sécurité et insécurité linguistique chez les libanais francophones ». Insécurité linguistique dans les communautés francophones périphériques. Dir. M. Francard. Louvain-La-Neuve : Fondation U de Belgique et de la Communauté française de Belgique, Service de la langue française, 1994. 35-44.

Coste, Daniel, Daniel Moore et Geneviève Zarate. Compétence plurilingue et pluriculturelle. Vers un Cadre européen commun de référence pour l'enseignement et l'apprentissage des langues vivantes. Strasbourg : Conseil de l'Europe, 2009 (1997).

Dolbec, André et John Clement. «La recherche-action». Introduction à la recherche en éducation. Dir. Thierry Karsenti et Lorraine Savoie-zacj. Canada, 2000. 199-224.

European Center for Modern Languages. «Framework of reference for pluralistic approaches to languages and cultures », 2007. http://carap.ecml.

Hawkins, Eric. Awareness of Language. An introduction. Cambridge : Cambridge UP, 1994.

Labov, William. Sociolinguistic Patterns. Philadelphie : U of Pennsylvania P, 1972. Trad. fr. Sociolinguistique. Paris : Minuit, 1976.

La lettre de l'ACEDIFLE. «Une autre voie pour les langues à l'école primaire : faire de la diversité un instrument d'éducation », 2002.

Laudet, Véronique. «Construction identitaire et multilinguisme : une étude de cas ». U Grenoble Stendhal 3. Mémoire. 2003.

Puren, Christian. «Le Cadre européen commun de référence et la réflexion méthodologique en didactique des langues-cultures : un chantier à reprendre ». Site de l'APLV, 2006.

Said, Edward. «Faut-il préférer le classique au dialectal ? La langue arabe, la Rolls et la Volkswagen ». Le monde diplomatique (août 2004).

Tahan, Mireille. «L'enseignement du et en français au Liban dans les établissements privés francophones ». Faire vivre les identités : nn parcours en francophonie. Dir. Patrick Chardenet et Jean-Pierre Cuq. Paris: Agence U de la francophonie, Archives contemporaines, 2010. 45-49.

\section{Recherches menées au Liban}

Grappe, Isabelle. «Apprentissage/multilinguisme et identité des enseignants au Liban». U Stendhal, Grenoble 3. DEA. 2002.

---. « Conception et évaluation d'une formation de formateurs au français sur objectifs spécifiques à partir d'une approche sociolinguistique ». U de Grenoble. Thèse. 2010.

Raychouni, Lama. «Contribution d'un dispositif de didactique intégrée à la prise de parole des élèves en arabe classique ». U Saint Joseph. Master. 2013. 
Yaghi, Ghinwa. «Les représentations des langues et de leur enseignement/apprentissage chez les enseignants de langues arabe, français, anglais ». U Saint Joseph, Liban. Master. 2013

NOTES

1 «Evlang »est «un programme d'innovation et de recherches pédagogiques consacré à une approche originale des langues à l'école primaire. » (19). Ce programme qui s'inscrit dans celui de l'éveil aux langues a été lancé par une équipe de trente chercheurs européens.

${ }^{2}$ La didactique intégrée des langues vise à aider l'apprenant à établir des liens entre un nombre limité de langues, celles dont on recherche l'apprentissage dans un cursus scolaire, selon l'idée centrale pour les approches plurielles d'un appui sur le connu pour aborder le moins connu (CARAP).

${ }^{3}$ Conseil de 1'Europe. «Cadre des Approches Plurielles des langues et des Cultures ».

${ }^{4}$ Jacqueline Billiez, professeur émérite, U de Grenoble

${ }^{5}$ Le Cadre européen commun de référence pour les langues - Apprendre, Enseigner, Évaluer est un document publié par le Conseil de l'Europe en 2001, qui définit des niveaux de maîtrise d'une langue étrangère en fonction de savoir-faire dans différents domaines de compétence.

${ }^{6}$ Cette approche didactique met en œuvre des activités d'enseignement-apprentissage qui impliquent à la fois plusieurs variétés linguistiques et culturelles Cadre de référence pour les approches plurielles des langues et des cultures, p. 7.

${ }^{7}$ Ce contrat didactique a été présenté et appliqué lors du déroulement des cinq séances du dispositif d'éveil aux langues et de didactique intégrée.

${ }^{8}$ «Evlang » est « un programme d'innovation et de recherches pédagogiques consacré à une approche originale des langues à l'école primaire. »(Candelier 19). Ce programme qui s'inscrit dans celui de l'éveil aux langues a été lancé par une équipe de 30 chercheurs européens.

${ }^{9}$ Raychouni,L « contribution d'un dispositif de didactique intégrée à la prise de parole des élèves en arabe classique », Master, U Saint Joseph 